\title{
Pyrrole modified biomass derived hierarchical porous carbon as high performance symmetrical supercapacitor electrodes
}

\author{
Keliang Wang ${ }^{\mathrm{a}}$, Ming $\mathrm{Xu}^{\mathrm{c}}$, Zhengrong $\mathrm{Gu}^{* a}$, Phil Ahrenkiel ${ }^{\mathrm{d}}$, Joun Lee ${ }^{\mathrm{e}}$, William Gibbons ${ }^{\mathrm{f}}$, \\ Jason Croat ${ }^{\mathrm{f}}$, Qihua Fan $^{*} b$ \\ a Agricultural and Biosystems Engineering Department, South Dakota State University, Brookings, SD 57007, United States \\ b Electrical Engineering and Computer Science Department, South Dakota State University, Brookings, SD 57007, United States \\ c PetroChina Lanzhou Petrochemical Company, Lanzhou, Gansu, 730060, China \\ d Nanoscience and Nanoengineering Department, South Dakota School of Mines and Technology, Rapid City, SD 57701 United States \\ e Department of Chemistry ,University of Iowa, Iowa City, IA, 52242, United States
}

f Biology and Microbiology Department, South Dakota State University, Brookings, SD 57007, United States

\section{Abstract:}

Nitrogen-doped hierarchical porous carbon has been prepared via a direct carbonization of pyrrole modified biomass for using as electrochemical double layers capacitance electrodes materials. The hierarchical porous carbon features high specific area $\left(2959 \mathrm{~m}^{2} \mathrm{~g}^{-1}\right)$ and porosity, which originated from the etching, gasification and intercalation effect during heat treatment. Based on this unique porous structure, specific capacitance of $270 \mathrm{~F} \mathrm{~g}^{-1}$ at low current density of $0.1 \mathrm{~A} \mathrm{~g}^{-1}$ and $232 \mathrm{~F} \mathrm{~g}^{-1}$ at high current density of $10 \mathrm{~A} \mathrm{~g}^{-1}$ was obtained in $6 \mathrm{M} \mathrm{KOH}$ electrolyte. Excellent cycles durability was obtained after initial charge/discharge, the high specific capacitance remained nearly unchanged for total 4,000 cycles tested over a broad current density ranging from $0.1 \mathrm{~A} \mathrm{~g}^{-1}$ to $10 \mathrm{~A}$. This study indicates that hierarchical porous carbon derived from pyrrole modified biomass is a promising electrode material for symmetric supercapacitors.

Keywords: biomass, N-doped, hierarchical porous carbon, symmetric supercapacitor

\footnotetext{
${ }^{\text {a }}$ Z R. Gu Tel./fax: + 1605688 5372. Email address: zhengrong.gu@sdstate.edu (Z R. Gu)

${ }^{\text {b }}$ Q H. Fan Tel./fax: + 1605 688-5910. Email address: qihua.fan@sdstate.edu (Q H. Fan)
} 


\section{Introduction}

28 Supercapacitors are promising energy storage devices due to their long life cycle, high power and energy density, and excellent stability. Based on their working principles, supercapacitors can be divided into two major types: pesudocapacitors and electrochemical double layer capacitors (EDLCs). Pesudocapacitors store energy storage via faradic redox reactions where occurs on the surface of electrode materials immersed into an electrolyte [1]. The EDLCs, also referred as symmetric supercapacitors, store energy via electric charge accumulation at the electrode/electrolyte interface [2]. Pesudocapacitors use metal oxides as electrode materials.

35 Among the available metals oxides, $\mathrm{RuO}_{2}$ displayed excellent capacitance performance but are expensive [3]. To overcome this drawback, alternative metal oxides (such as $\mathrm{NiO}, \mathrm{MnO}_{2}, \mathrm{Co}_{3} \mathrm{O}_{4}$

37 and $\mathrm{WO}_{3}$ [4-7]) have been developed. However, the capacitance is still limited by poor conductivity, low power density and poor rate capability [8]. On the other hand, carbon has been used as electrode material for EDLCs due to its stable physicochemical properties, good conductivity, low cost, and abundant source. A well accepted opinion is that carbon material

41 with high specific surface area (SSA) and porosity is particularly suitable for EDLC electrodes.

42 The high SSA can provide enough accessible contact area between the electrolyte and electrode

43 surface, and the porous structure can serve as reservoir and facilitate the electrolyte diffusion

44 from exterior into interior of the electrode material. Porous carbon electrodes have been 45 demonstrated with very high specific capacitance in carbon EDLCs [9, 10]. As a result, 46 considerable effort has been made to create ideal porous carbon for EDLCs. Among those 47 reported approaches, $\mathrm{KOH}$ activation under pyrolysis is considered an efficient way because the 48 chemical activation produces a porous network by etching the physical activation further 49 develops the pores through gasification, and the metallic $\mathrm{K}$ expands the carbon lattice by 
50 intercalation effect [11, 12]. Based on these synergistic effects, various activated carbon

51 materials with large SSA and high porosity have been developed for EDLCs.

52 However, the specific capacitance achieved using porous carbon was still not high enough. The

53 relatively low conductivity of porous carbon appeared to be one limiting factor. It was found

54 recently that introducing heteroatom, such as $\mathrm{N}, \mathrm{B}$, and $\mathrm{S}$ [13-15], was a promising way to

55 improve the EDLCs performance. Among these dopants, $\mathrm{N}$ exhibited more advantages than

56 others because it not only improved conductivity, but also enhanced surface wettability and

57 induced pseudo-capacitance [16-18]. In addition, the cost of activated carbon is also a big

58 concern for mass production. Compared with conventional carbon nanomaterials (carbon

59 nanotubes, carbon fibers, and graphene), biomass-derived activated carbon is very attractive

60 because of its availability, low cost and sustainability [19-22]. As a consequence, using N-

61 modified biomass as carbon precursor is expected to be a good strategy to prepare high

62 performance EDLCs.

63 Herein, the authors report a kind of hierarchical activated carbon derived from pyrrole modified

64 fungus via efficient $\mathrm{KOH}$ activation. The $\mathrm{KOH}$ induced physical and chemical activation, created

65 hierarchical porous structure with high SSA. Meanwhile, N composite was converted into

66 beneficial $\mathrm{N}$ species during the pyrolysis process. The activated carbon was then used to prepare

67 electrodes for EDLCs, which exhibited high specific capacitance, excellent stability, and long 68 life time.

\section{Experimental}

\subsection{Preparation of activated carbon}

71 Dried fungus (3 g), prepared from pullulans, was mixed with pyrrole $(1.5 \mathrm{ml})$ and $30 \mathrm{ml}$ ethanol

72 in a beaker, followed by stirring at $80^{\circ} \mathrm{C}$ until the ethanol was evaporated. Then the mixture was 
73 heated at $400{ }^{\circ} \mathrm{C}$ for one hour with a heating rate of $10^{\circ} \mathrm{C} \min ^{-1}$ in a muffle furnace (1100 box

74 furnace, Lindberg/Blue M, Thermo Scientific Inc.) under the protection of nitrogen (flow rate

75 was $96 \mathrm{ml} \mathrm{min}^{-1}$ ). After the precarbonized material was cooled to room temperature, it was

76 mixed with potassium hydroxide (KOH, Fisher Scientific Inc.) in a mass ratio of 1:3 in a steel

77 crucible that contained $30 \mathrm{~mL}$ deionized water. The crucible was placed in an oven and dried at

$78110{ }^{\circ} \mathrm{C}$ for $24 \mathrm{~h}$. Then, the crucible was transferred into a muffle and activated at $800{ }^{\circ} \mathrm{C}$ for $1 \mathrm{~h}$

79 with a heating rate of $10^{\circ} \mathrm{C} \mathrm{min}^{-1}$ under $\mathrm{N}_{2}$ protection (flow rate was $96 \mathrm{ml} \mathrm{min}^{-1}$ ). Afterwards,

80 the carbonized solids were washed with $30 \mathrm{~mL} 0.1 \mathrm{~mol} \mathrm{~L}^{-1} \mathrm{HCl}$ (Fisher Scientific Inc.) at $110{ }^{\circ} \mathrm{C}$

81 for $1 \mathrm{~h}$ in a $60 \mathrm{~mL}$ polytetrafluoroethylene (PTFE) autoclave to remove the residual $\mathrm{KOH}$ and

82 impurities. Finally, the carbon solids was washed several times with deionized water until the $\mathrm{pH}$

83 stabilized at 7 . The material was then dried at $105{ }^{\circ} \mathrm{C}$ overnight in an oven. The carbonized

84 samples were denoted as KPF.

85 For comparison, samples prepared using the same procedure but without $\mathrm{KOH}$ were denoted as

$86 \mathrm{PF}$, and raw fungus directly carbonized with the same procedure as PF but without adding

87 pyrrole was denoted as F.

882.2 Preparation of electrodes

89 Electrodes were prepared by mixing the F, PF or KPF (80wt\%) with acetylene black (10wt\%) 90 and PTFE (10 wt\%), and then pressing onto a nickel foam (EQ-bcnf-16m, MTI Corp.) of $1 \mathrm{~cm}^{2}$

91 surface area. The electrodes were dried at $60{ }^{\circ} \mathrm{C}$ for $12 \mathrm{~h}$ in an oven. Afterwards, a sandwich

92 structure was formed by placing two pieces of microporous Polypropylene (PP) separator

93 celgard-3501 between two electrodes in a coin cell 2032 type system. Finally, the cell was

94 pressed under a pressure of $1,000 \mathrm{~kg} \mathrm{~cm}^{-2}$ to finish the assembly. 
96 The surface analysis was performed on a Kratos Axis Ultra high performance X-ray

97 photoelectron spectroscopy (XPS) (Kratos Analytical Ltd.). For high resolution data, the lowest

98 binding-energy $\mathrm{C}$ 1s peak was set at $285.0 \mathrm{eV}$ and used as the reference for other elements.

99 Raman spectra were obtained on a Horiba LABRam confocal Raman microscopy system with 100 excitation wavelength of $532 \mathrm{~nm}$ from a diode pumped solid state laser. Isothermal adsorption 101 analysis with dry $\mathrm{N}_{2}$ was carried out at $77 \mathrm{~K}$ (liquid nitrogen bath), using ASAP2020 102 (Micromeritics Inc.) micropore analyzer. The specific surface area was determined by the 103 Brunauer-Emmett-Teller (BET) method and the pore size distribution was calculated according 104 to the density functional theory (DFT) method using non-local density functional theory 105 (NLDFT) analysis for carbon with slit pore model. A transmission electron microscope (TEM) 106 (JEM-2100 LaB6, JEOL) equipped with energy-dispersive X-ray spectroscopy (EDX) was used 107 to study the morphology and microstructure at an acceleration voltage of $200 \mathrm{kV}$.

\subsection{Electrochemical characterization:}

109 Cyclic voltammetry (CV) and electrochemical impedance spectroscopy tests were measured on 110 an electrochemical work station (SP-150, BioLogical, France) in $6 \mathrm{~mol} \mathrm{~L}^{-1} \mathrm{KOH}$ electrolyte. The 111 galvanostatic charge/discharge curves were obtained from a battery test system (BTS series, 112 NEWARE, China).

\section{3. Results and discussion}

114 To observe the morphology of F, PF and KPF, TEM and SEM were conducted (Fig. 1). As can 115 be seen (Fig. 1a and b), samples F and PF were strongly aggregated chunks. On contrast, 116 hierarchical and porous structure clearly appeared in the KPF (Fig. 1c and d). In addition, the 117 pore structures was examined by high resolution TEM (Fig. 1e and f), revealing large amount of 
118 pores with different sizes. Such a porous network was believed to be originated from $\mathrm{KOH}$ 119 activation during the pyrolysis process. As mentioned above, synergistic effects, including 120 etching, gasification and intercalation effect were responsible for pore network formation, 121 porosity enhancement, and lattice expansion, respectively [11]. Such hierarchical morphology 122 could provide large SSA. Moreover, from electrochemical perspective, a smaller resistance and 123 shorter diffusion pathways were created by channels connected with pores of different sizes to 124 facilitate the ion transport [9, 10], and were expected to lead to an improvement in capacitance 125 performance as EDLCs electrodes.

126 To verify the speculation on high SSA and pore structure observed in TEM images, $\mathrm{N}_{2}$ 127 adsorption/desorption isotherm measurement was performed (Fig. 2a). The plot showed a typical 128 type I isotherm according to IUPAC classification and high SSA up to $2959 \mathrm{~m}^{2} \mathrm{~g}^{-1}$. The inset in 129 Fig. 2a depicted pore size distribution of KPF. A strong peak located at $15 \mathrm{~nm}$ was showed, 130 indicating the pore network mainly consisted of mesopores, while micropore and macropore took 131 a small percentage. Among these three kinds of pores, mesopores were believed to offer lower 132 ion-transport resistance via mesoporous channels [10, 23-26]. Micropores would impose a long 133 diffusion distance ( $>5 \mathrm{~mm}$ ) and a high ion-transport resistance led to a large electrode-potential 134 drop $\left(I R_{d r o p}\right)$ and a low ion-accessible surface area at high current values [27]. Macropores 135 played a role as ion-buffering reservoirs, which would lead to a decreased diffusion distance[10]. 136 Raman spectrum was used to characterize the carbon structures of KPF. As shown in Fig. 2b, 137 two characteristic peaks located at around $1340 \mathrm{~cm}^{-1}$ and $1580 \mathrm{~cm}^{-1}$ were presented, which were 138 assigned to $D$-band and $G$-band, respectively [28]. The $D$-band was related with the vibration of 139 the carbon atoms with dangling bonds in plane terminations of the disordered structure, while $G$ 140 band reflected the vibration of $s p^{2}$-bonded carbon atoms [28, 29]. 
141 XPS was employed to characterize the carbon, oxygen and nitrogen chemical states in the KPF 142 sample (Fig. 3). As shown in Fig. 3a, the high resolution C 1s spectrum was deconvoluted 143 into five peaks at binding energy of $c a .285 .2,286.3,287.5,289.1$ and $291.0 \mathrm{eV}$, corresponding 144 to $\mathrm{C}-\mathrm{C}, \mathrm{C}-\mathrm{O} / \mathrm{C}-\mathrm{OH}, \mathrm{C}=\mathrm{O} / \mathrm{C}-\mathrm{N}, \mathrm{COOH}$, and $\mathrm{CO}_{2} / \mathrm{CO}_{3}{ }^{2-}$ bonds and functional groups, 145 respectively[30, 31]. In addition, high resolution spectrum of O1s was deconvoluted into three 146 peaks (Fig. 3b), which were assigned to O-I, O-II and O-III at ca. $531 \mathrm{eV}, 532 \mathrm{eV}$, and $535 \mathrm{eV}$, 147 respectively. The three species $\mathrm{O}$ represented $\mathrm{C}=$ bond quinone type groups for O-I, C-OH phenol 148 groups and/or C-O-C ether groups for O-II, and chemisorbed oxygen for $\mathrm{COOH}$ carboxylic 149 groups and/or water for O-III, respectively. However, it was believed that only O-I represented 150 the pseudo-capacitance effect[32]. Besides, two peaks were detected at binding energy ca. 398.9 151 and $401.2 \mathrm{eV}$ for $\mathrm{N}$ species, and were attributed to pyridinic- $\mathrm{N}$, and quaternary- $\mathrm{N}$, respectively 152 (Fig. 3c). As reported previously[16, 17], $\mathrm{N}$ introduction would improve the conductivity and 153 pyridinic-N could lead to great pseudocapacitance effect, both of which would enhance the 154 capacitance performance.

155 To verify the performance of prepared electrode materials, electrochemical measurements were 156 employed. As presented in Fig. 4a c and e, the CV tests were conducted at various scanning rates 157 ranging from 5 to $300 \mathrm{mV} \mathrm{s}^{-1}$ for $\mathrm{F}, \mathrm{PF}$ and $\mathrm{KPF}$, respectively. The $\mathrm{F}$ and PF electrodes exhibited 158 distorted rectangular shapes even at low scanning rate, while typical rectangular shape was 159 observed for KPF electrode. In addition, the KPF electrodes retained rectangular shape CV 160 curves when the scanning rate increased from $5 \mathrm{mV} \mathrm{s}^{-1}$ to high $300 \mathrm{mV} \mathrm{s}$. These results 161 indicated the high capacitive performance with rapid charging-discharging characteristics due to 162 efficient charge transfer and electrolyte diffusion within the porous KPF[22]. 
163 The capacitive performance was further evaluated using the following equation[33] in the two164 electrode system:

$$
C=\frac{2 I \Delta t}{m \Delta v}
$$

166 where $\mathrm{C}$ is the specific capacitance, $I$ is the charge/discharge current, $\Delta t$ is the corresponding 167 charge or discharge time, $m$ is the mass of active materials on single electrode, and $\Delta v$ is the total 168 corresponding potential change. As can be observed in Fig. 4a and c, the specific capacitance 169 was only around $60 \mathrm{~F} \mathrm{~g}^{-1}$ for both $\mathrm{F}$ and PF electrodes. On contrast, high specific capacitance of $170245,243,238,234,233,223$ and $204 \mathrm{~F} \mathrm{~g}^{-1}$ was obtained at scanning rate of 5, 10, 20, 50, 100, 171200 and $300 \mathrm{mV} \mathrm{s}^{-1}$ for KPF, respectively. Combining the physical measurements results, it was 172 deduced that the high specific capacitance could be attributed to large specific surface area, 173 hierarchical porous structure, and high degree of graphitization of KPF.

174 Galvanostatic charge/discharge measurements were performed at different current densities 175 ranging from 0.1 to $10 \mathrm{~A} \mathrm{~g}^{-1}$ for $\mathrm{F}, \mathrm{PF}$ and KPF (Fig. 4b, d and f). Differing from the comparable 176 symmetric triangle shape and an obvious $I R_{\text {drop }}$ for $\mathrm{F}$ and PF electrodes, KPF displayed a 177 symmetric triangle shape, suggesting a very small internal resistance and less energy being 178 wasted during charge/discharge process[27].

179 Fig. 5a shows Nyquist plots that was obtained from KPF electrodes in $6 \mathrm{~mol} \mathrm{~L}^{-1} \mathrm{KOH}$ electrolyte. 180 In general, the total resistance $(\sim 2.5 \Omega)$ consisted of bulk electrolyte resistance $\left(R_{1}\right)$, interfacial 181 impedance between electrode and bulk solution $\left(R_{2}\right)$, and the impedance associated with intra182 particle pores $\left(R_{3}\right)$, corresponding to $x$-intercept at the highest frequency region, semicircle at the 183 middle frequency region and spike at the low frequency region, respectively [34]. $R_{1}$ and $R_{2}$, 184 related to the electrolyte solution, while $R_{3}$ depended on both electrode materials and electrolytes. 
185 This result was in consistent with the expected low resistance, hierarchical structure and high SSA confirmed above.

187 As another significant parameter of EDLCs performance, cycling life of KPF electrodes was 188 recorded in $6 \mathrm{~mol} \mathrm{~L}^{-1} \mathrm{KOH}$ electrolyte at different current density ranging from 0.1 to $10 \mathrm{~A} \mathrm{~g}^{-1}$. 189 As shown in Fig. 5b, large specific capacitance of 270, 243, 235, 233 and $232 \mathrm{~F} \mathrm{~g}^{-1}$ were 190 obtained at current density of $0.1,0.5,1.0,5.0$ and $10.0 \mathrm{~A} \mathrm{~g}^{-1}$ for each individual 1,000 cycles, 191 respectively. Apart from a little bit degradation in the first 1,000 cycles at current density of 0.1 $192 \mathrm{~A} \mathrm{~g}^{-1}$, excellent cycle stability (almost 100\% retention) was displayed, which demonstrated the 193 excellent stability of KPF as electrode materials for EDLCs.

\section{3. Conclusion}

195 N-doped hierarchical porous carbon has been successfully prepared from pyrrole modified 196 abundant fungus as high-performance electrodes for EDLCs. The prepared KPF carbon exhibited 197 extraordinarily large SSA (2959 $\left.\mathrm{m}^{2} \mathrm{~g}^{-1}\right)$ and porosity, which served as ion diffusion channels and 198 electrolyte reservoir contributed to capacitance enhancement. Large specific capacitance of 270 $199 \mathrm{~F} \mathrm{~g} \mathrm{~g}^{-1}$ at low current density of $0.1 \mathrm{~A} \mathrm{~g}^{-1}$ and $232 \mathrm{~F} \mathrm{~g}^{-1}$ at high current density of $10 \mathrm{~A} \mathrm{~g}^{-1}$ was 200 obtained in $6 \mathrm{M} \mathrm{KOH}$ solution. In addition, excellent cycle stability for continuous 1,000 cycles 201 at different current densities was achieved for assembled EDLCs. This work demonstrated the 202 pyrrole modified biomass derived porous carbon was a promising electrode material for electric 203 energy storage.

\section{Acknowledgement}

205 This research was funded by: 1) China Scholarship Council; 2) NSF MRI award \#1427888; 3) NSF EPSCoR Track II Dakota BioCon Center \#1330840; 4) NSF award \#1462389; 5) NSF 
207 award \#1536209; 6) NSF CHE-0840507. 7) North Central Regional Sun Grant Center at South

208 Dakota State University through a grant provided by the US Department of Energy Bioenergy

209 Technologies Office under award number DE-FG36-08G088073.

210 References

211 [1] Fang J, Li M, Li Q, Zhang W, Shou Q, Liu F, et al. Microwave-assisted synthesis of CoAl-

212 layered double hydroxide/graphene oxide composite and its application in supercapacitors.

213 Electrochim Acta 2012;85:248-55.

214 [2] Lei C, Markoulidis F, Ashitaka Z, Lekakou C. Reduction of porous carbon/Al contact 215 resistance for an electric double-layer capacitor (EDLC). Electrochim Acta 2013;92:183-7.

216 [3] Min M, Machida K, Jang JH, Naoi K. Hydrous $\mathrm{RuO}_{2}$ /carbon black nanocomposites with 3D

217 porous structure by novel incipient wetness method for supercapacitors. J Electrochem Soc 218 2006;153:A334-A8.

219 [4] Xia X-h, Tu J-p, Mai Y-j, Wang X-l, Gu C-d, Zhao X-b. Self-supported hydrothermal 220 synthesized hollow $\mathrm{Co}_{3} \mathrm{O}_{4}$ nanowire arrays with high supercapacitor capacitance. J Mater Chem $221 \quad$ 2011;21:9319-25.

222 [5] Zhang Y, Li G-y, Lv Y, Wang L-z, Zhang A-q, Song Y-h, et al. Electrochemical 223 investigation of $\mathrm{MnO}_{2}$ electrode material for supercapacitors. Int $\mathrm{J}$ Hydrogen Energ $224 \quad 2011 ; 36: 11760-6$.

225 [6] Chen Y, Huang Z, Zhang H, Chen Y, Cheng Z, Zhong Y, et al. Synthesis of the 226 graphene/nickel oxide composite and its electrochemical performance for supercapacitors. Int J 227 Hydrogen Energ 2014;39:16171-8. 
228 [7] Wei H, Yan X, Wang Q, Wu S, Mao Y, Luo Z, et al. Electrochemical properties and 229 electrochromic behaviors of the sol-gel derived tungsten trioxide thin films. Energy Enviro 230 Focus 2013;2:112-20.

231 [8] Lu Y, Zhang F, Zhang T, Leng K, Zhang L, Yang X, et al. Synthesis and supercapacitor 232 performance studies of $\mathrm{N}$-doped graphene materials using o-phenylenediamine as the double-N 233 precursor. Carbon 2013;63:508-16.

234 [9] Xu F, Cai R, Zeng Q, Zou C, Wu D, Li F, et al. Fast ion transport and high capacitance of 235 polystyrene-based hierarchical porous carbon electrode material for supercapacitors. J Mater 236 Chem 2011;21:1970-6.

237 [10] Wang DW, Li F, Liu M, Lu GQ, Cheng HM. 3D aperiodic hierarchical porous graphitic 238 carbon material for high-rate electrochemical capacitive energy storage. Angew Chem $239 \quad 2008 ; 120: 379-82$.

240 [11] Wang J, Kaskel S. KOH activation of carbon-based materials for energy storage. J Mater 241 Chem 2012;22:23710-25.

242 [12] Chen X, Wang H, Yi H, Wang X, Yan X, Guo Z. Anthraquinone on porous carbon 243 nanotubes with improved supercapacitor performance. J Phys Chem C 2014;118:8262-70.

244 [13] Seredych M, Bandosz TJ. S-doped micro/mesoporous carbon-graphene composites as 245 efficient supercapacitors in alkaline media. J Mater Chem A. 2013;1:11717-27.

246 [14] Guo H, Gao Q. Boron and nitrogen co-doped porous carbon and its enhanced properties as 247 supercapacitor. J Power Sources 2009;186:551-6.

248 [15] Chen L-F, Zhang X-D, Liang H-W, Kong M, Guan Q-F, Chen P, et al. Synthesis of 249 nitrogen-doped porous carbon nanofibers as an efficient electrode material for supercapacitors. 250 ACS nano 2012;6:7092-102. 
251 [16] Yang X, Wu D, Chen X, Fu R. Nitrogen-enriched nanocarbons with a 3-D continuous 252 mesopore structure from polyacrylonitrile for supercapacitor application. J Phys Chem C $253 \quad 2010 ; 114: 8581-6$.

254 [17] Tan Y, Xu C, Chen G, Liu Z, Ma M, Xie Q, et al. Synthesis of ultrathin nitrogen-doped 255 graphitic carbon nanocages as advanced electrode materials for supercapacitor. ACS Appl Mater 256 \& Inter 2013;5:2241-8.

257 [18] Wei H, Gu H, Guo J, Wei S, Guo Z. Electropolymerized polyaniline nanocomposites from 258 multi-walled carbon nanotubes with tuned surface functionalities for electrochemical energy 259 storage. J Electrochem Soc 2013;160:G3038-G45.

260 [19] Ding J, Wang H, Li Z, Cui K, Karpuzov D, Tan X, et al. Peanut shell hybrid sodium ion 261 capacitor with extreme energy-power rivals lithium ion capacitors. Energ \& Environ Sci $262 \quad 2015 ; 8: 941-55$.

263 [20] Fan Y, Liu P, Zhu B, Chen S, Yao K, Han R. Microporous carbon derived from acacia gum 264 with tuned porosity for high-performance electrochemical capacitors. Int J Hydrogen Energ 265 2015;40:6188-96.

266 [21] Jiménez-Cordero D, Heras F, Gilarranz MA, Raymundo-Piñero E. Grape seed carbons for 267 studying the influence of texture on supercapacitor behaviour in aqueous electrolytes. Carbon $268 \quad 2014 ; 71: 127-38$.

269 [22] Fan Y, Yang X, Zhu B, Liu P-F, Lu H-T. Micro-mesoporous carbon spheres derived from 270 carrageenan as electrode material for supercapacitors. J Power Sources 2014;268:584-90.

271 [23] Xia K, Gao Q, Jiang J, Hu J. Hierarchical porous carbons with controlled micropores and 272 mesopores for supercapacitor electrode materials. Carbon 2008;46:1718-26. 
273 [24] Liang Y, Wu D, Fu R. Preparation and electrochemical performance of novel ordered

274 mesoporous carbon with an interconnected channel structure. Langmuir 2009;25:7783-5.

275 [25] Yamada H, Moriguchi I, Kudo T. Electric double layer capacitance on hierarchical porous

276 carbons in an organic electrolyte. J Power Sources 2008;175:651-6.

277 [26] Xing W, Qiao S, Ding R, Li F, Lu G, Yan Z, et al. Superior electric double layer capacitors

278 using ordered mesoporous carbons. Carbon 2006;44:216-24.

279 [27] Wu Q, Xu Y, Yao Z, Liu A, Shi G. Supercapacitors based on flexible graphene/polyaniline 280 nanofiber composite films. ACS nano 2010;4:1963-70.

281 [28] Yang X, Li C, Wang W, Yang B, Zhang S, Qian Y. A chemical route from PTFE to 282 amorphous carbon nanospheres in supercritical water. Chem Commun 2004:342-3.

283 [29] Jawhari T, Roid A, Casado J. Raman spectroscopic characterization of some commercially 284 available carbon black materials. Carbon 1995;33:1561-5.

285 [30] Ding JD, Diao YF, Shen HG. Characters of nickel-loaded activated carbon fibers and 286 adsorption experiments of gaseous mercury. Adv Mater Res: Trans Tech Publ 2011. p.1211-4.

287 [31] Moreno-Castilla C, Lopez-Ramon M, Carrasco-Marın F. Changes in surface chemistry of 288 activated carbons by wet oxidation. Carbon 2000;38:1995-2001.

289 [32] Wang C, Sun L, Zhou Y, Wan P, Zhang X, Qiu J. P/N co-doped microporous carbons from 290 H 3 PO 4-doped polyaniline by in situ activation for supercapacitors. Carbon 2013;59:537-46.

291 [33] Wahid M, Parte G, Phase D, Ogale S. Yogurt: a novel precursor for heavily nitrogen doped 292 supercapacitor carbon. J Mater Chem A 2015;3:1208-15.

293 [34] Yoo HD, Jang JH, Ryu JH, Park Y, Oh SM. Impedance analysis of porous carbon electrodes 294 to predict rate capability of electric double-layer capacitors. J Power Sources 2014;267:411-20. 
Fig. 1 TEM images of F (a), PF (b) and KPF (c, e and f); SEM image of KPF (d).

Fig. 2 (a) $\mathrm{N}_{2}$ sorption-desorption isotherms of KPF. The insert in (a) shows pore size distribution; (b) Raman spectra of KPF.

Fig. 3 High resolution C 1s (a), O 1s (b) and N 1s (c) XPS spectra of KPF.

Fig. 4 CV curves of F (a), PF (c) and KPF (e) electrodes at various scanning rates ranging from 5 to $300 \mathrm{mV} \mathrm{s}^{-1}$; and galvanostatic charge/discharge curves of $\mathrm{F}(\mathrm{b}), \mathrm{PF}$ (d) and KPF (f) electrodes at different current densities ranging from 0.1 to $10 \mathrm{~A} \mathrm{~g}^{-1}$.

Fig. 5 (a) Nyquist plots of KPF electrodes; (b) Cycle life of KPF electrodes in $6 \mathrm{~mol} \mathrm{~L}^{-1} \mathrm{KOH}$ electrolyte at different current density ranging from 0.1 to $10 \mathrm{~A} \mathrm{~g}^{-1}$. 
Fig. 1

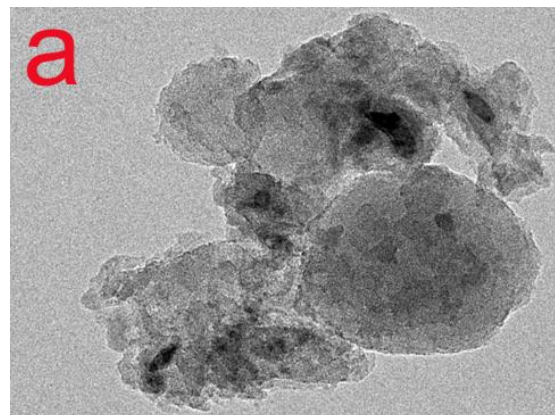

$100 \mathrm{~nm}$

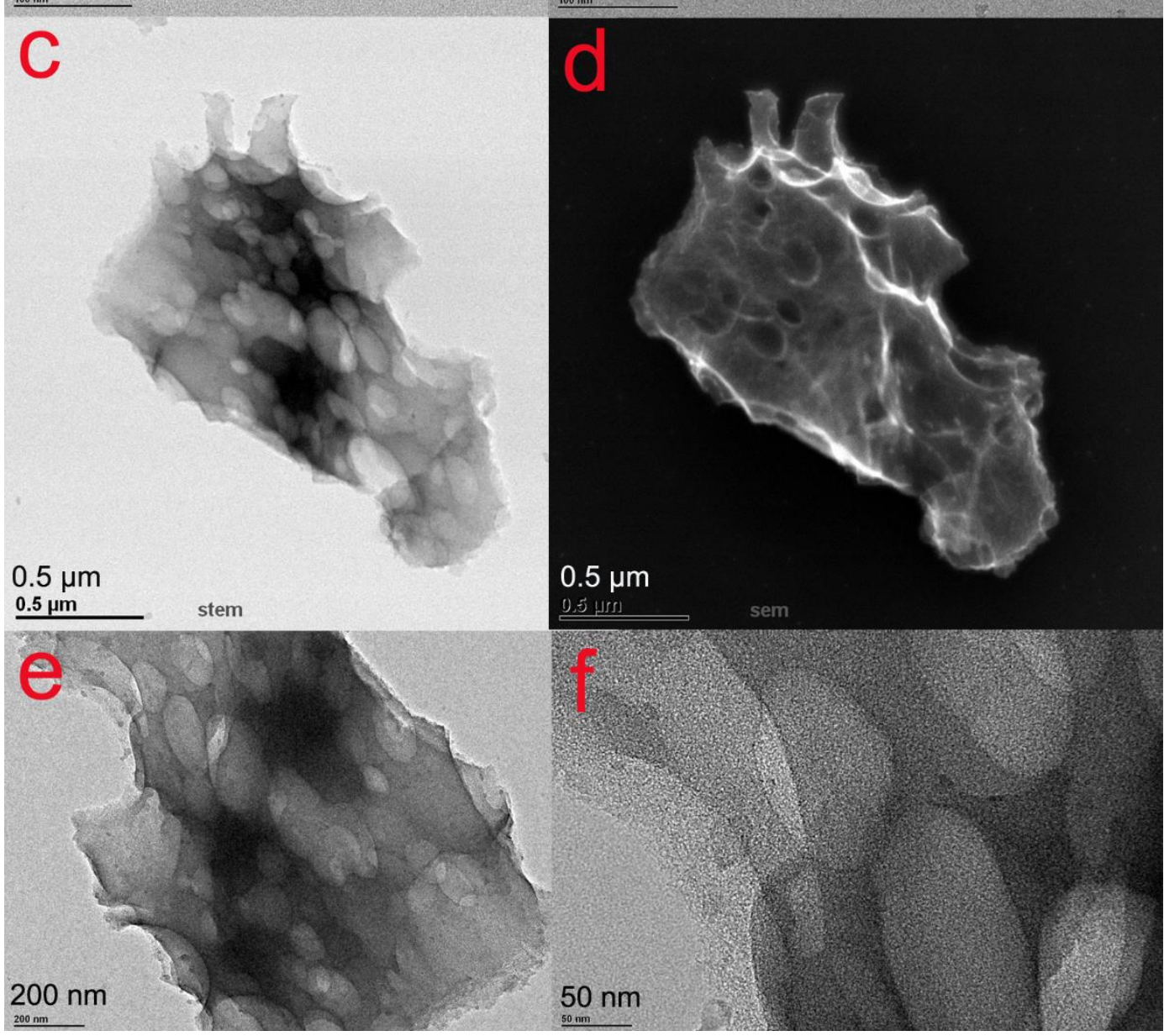


Fig. 2
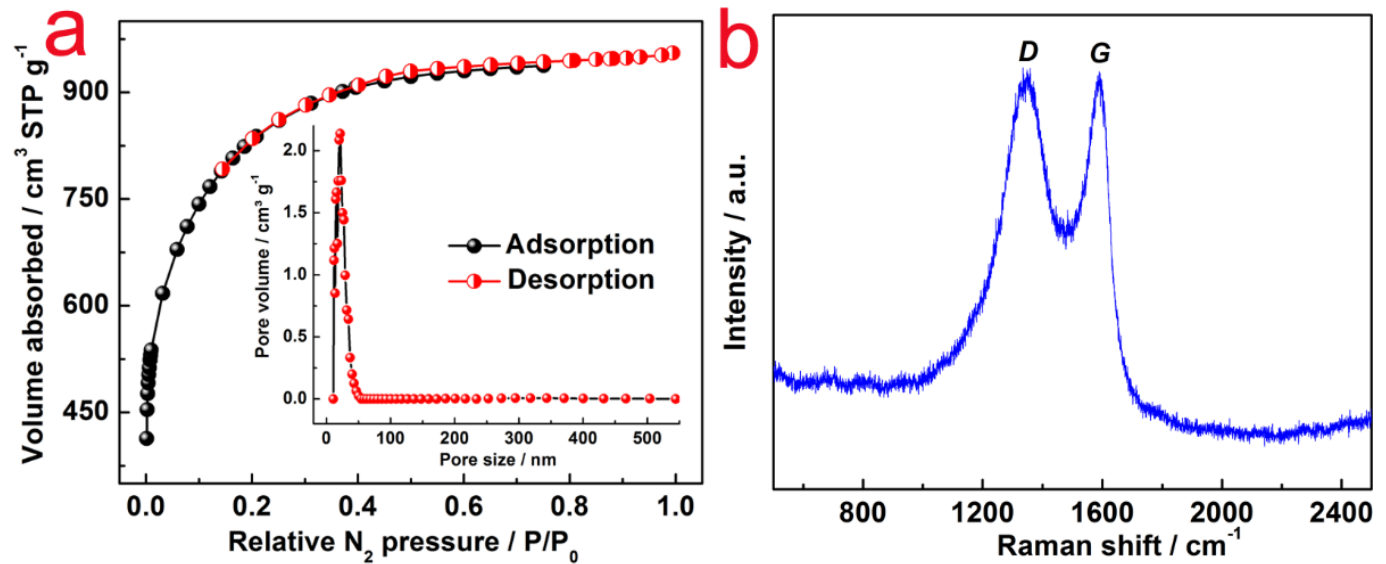
Fig. 3
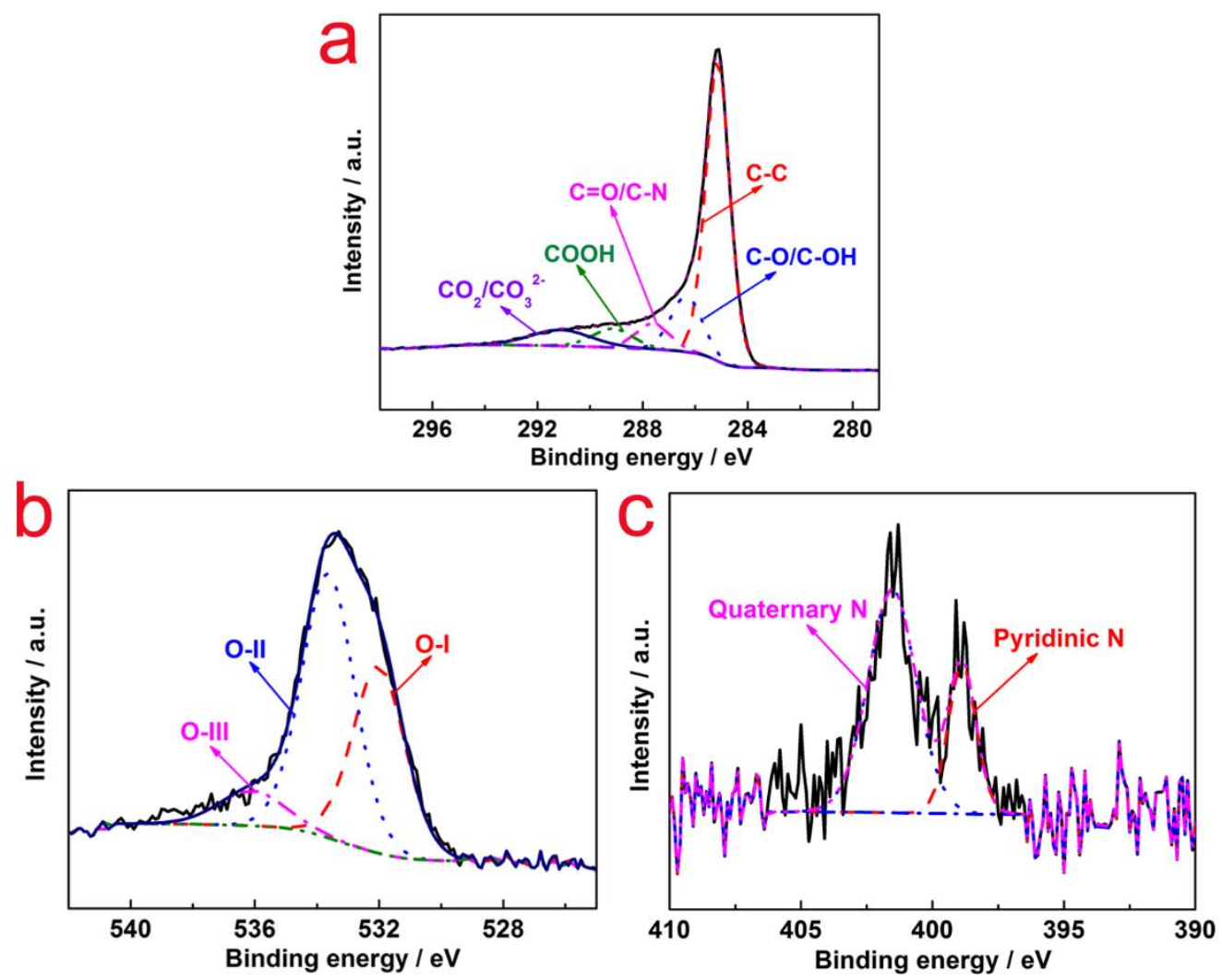
Fig. 4
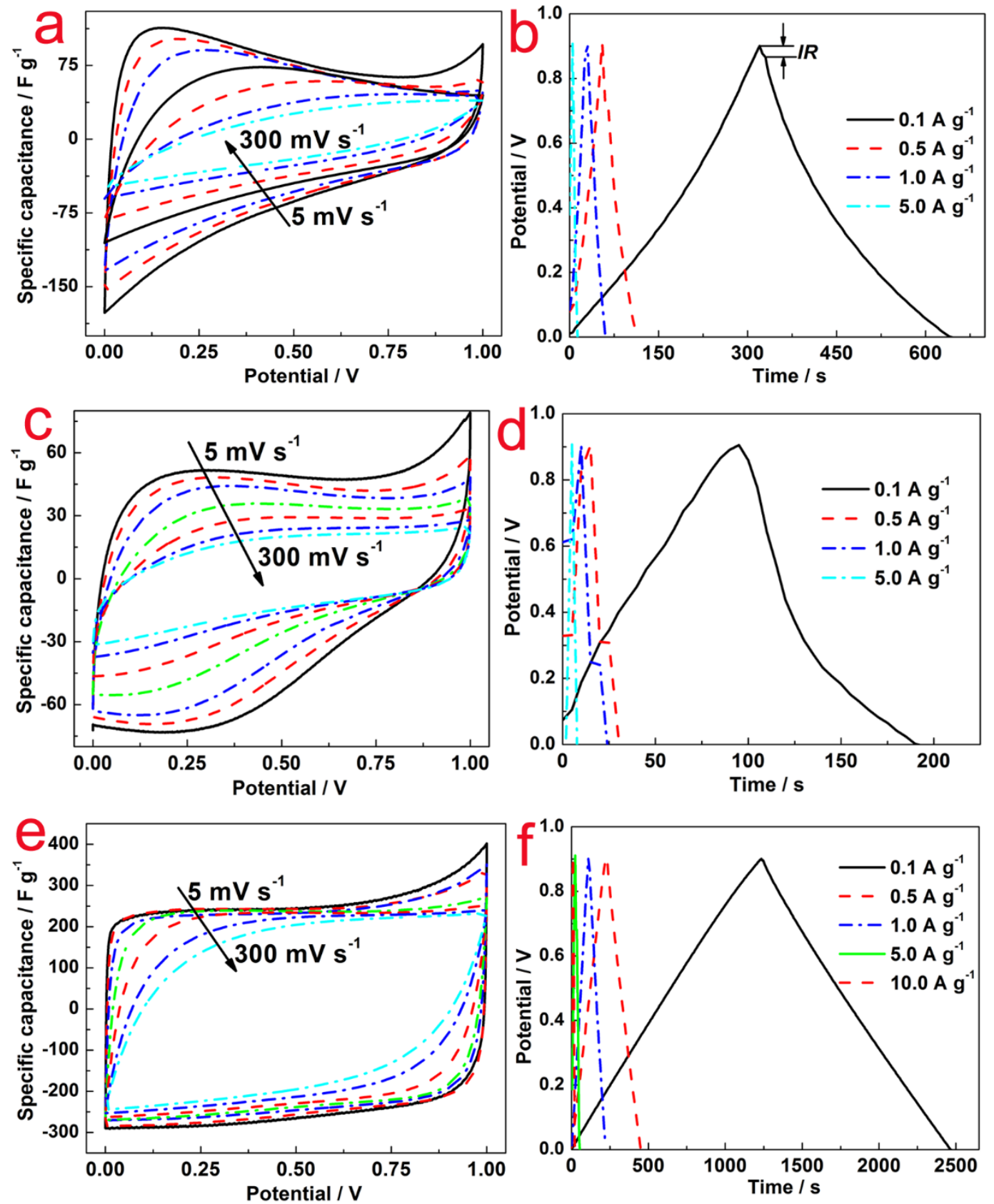
Fig. 5
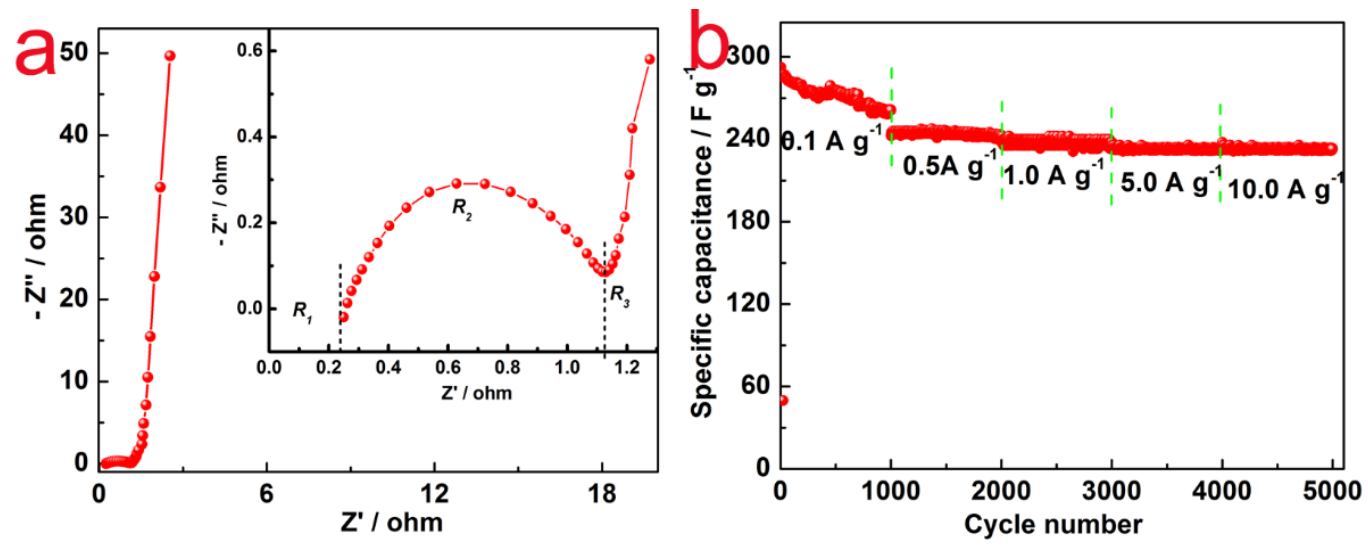


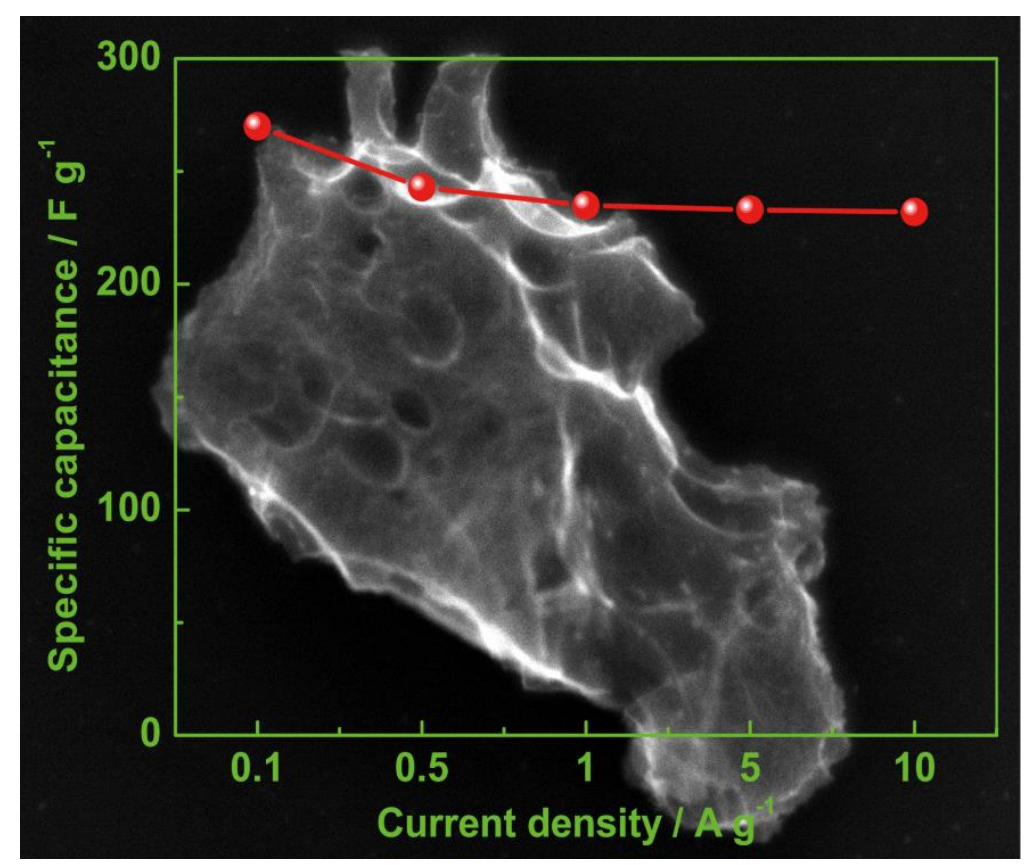

GRAPHICAL ABSTRACT 